\title{
Hydrodynamically Coupled Brownian Dynamics: A coarse-grain particle-based Brownian dynamics technique with hydrodynamic interactions for modeling self- developing flow of polymer solutions
}

V. R. Ahuja, J. van der Gucht, and W. J. Briels

Citation: The Journal of Chemical Physics 148, 034902 (2018); doi: 10.1063/1.5006627

View online: https://doi.org/10.1063/1.5006627

View Table of Contents: http://aip.scitation.org/toc/jcp/148/3

Published by the American Institute of Physics

\section{Articles you may be interested in}

Hydrodynamic relaxations in dissipative particle dynamics

The Journal of Chemical Physics 148, 034503 (2018); 10.1063/1.4986569

Efficient reactive Brownian dynamics

The Journal of Chemical Physics 148, 034103 (2018); 10.1063/1.5009464

Perspective: Dissipative particle dynamics

The Journal of Chemical Physics 146, 150901 (2017); 10.1063/1.4979514

Miscibility at the immiscible liquid/liquid interface: A molecular dynamics study of thermodynamics and mechanism

The Journal of Chemical Physics 148, 034707 (2018); 10.1063/1.5012506

An atomistic fingerprint algorithm for learning ab initio molecular force fields

The Journal of Chemical Physics 148, 034101 (2018); 10.1063/1.5008630

Calculation of a solid/liquid surface tension: A methodological study

The Journal of Chemical Physics 148, 034702 (2018); 10.1063/1.5008473

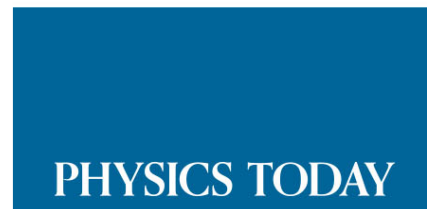




\title{
Hydrodynamically Coupled Brownian Dynamics: A coarse-grain particle-based Brownian dynamics technique with hydrodynamic interactions for modeling self-developing flow of polymer solutions
}

\author{
V. R. Ahuja, ${ }^{1,2,3, a)}$ J. van der Gucht, ${ }^{3}$ and W. J. Briels ${ }^{1,2,4, b)}$ \\ ${ }^{1}$ Computational Chemical Physics, Faculty of Science and Technology, University of Twente, \\ P.O. Box 217, 7500 AE Enschede, The Netherlands \\ ${ }^{2}$ MESA + Institute for Nanotechnology, University of Twente, P.O. Box 217, 7500 AE Enschede, \\ The Netherlands \\ ${ }^{3}$ Physical Chemistry and Soft Matter, Wageningen University, Building 124, Stippeneng 4, \\ 6708 WE, Wageningen, The Netherlands \\ ${ }^{4}$ Forschungszentrum Jülich, ICS 3, D-52425 Jülich, Germany
}

(Received 26 September 2017; accepted 21 December 2017; published online 16 January 2018)

\begin{abstract}
We present a novel coarse-grain particle-based simulation technique for modeling self-developing flow of dilute and semi-dilute polymer solutions. The central idea in this paper is the two-way coupling between a mesoscopic polymer model and a phenomenological fluid model. As our polymer model, we choose Responsive Particle Dynamics (RaPiD), a Brownian dynamics method, which formulates the so-called "conservative" and "transient" pair-potentials through which the polymers interact besides experiencing random forces in accordance with the fluctuation dissipation theorem. In addition to these interactions, our polymer blobs are also influenced by the background solvent velocity field, which we calculate by solving the Navier-Stokes equation discretized on a moving grid of fluid blobs using the Smoothed Particle Hydrodynamics (SPH) technique. While the polymers experience this frictional force opposing their motion relative to the background flow field, our fluid blobs also in turn are influenced by the motion of the polymers through an interaction term. This makes our technique a two-way coupling algorithm. We have constructed this interaction term in such a way that momentum is conserved locally, thereby preserving long range hydrodynamics. Furthermore, we have derived pairwise fluctuation terms for the velocities of the fluid blobs using the Fokker-Planck equation, which have been alternatively derived using the General Equation for the Non-Equilibrium Reversible-Irreversible Coupling (GENERIC) approach in Smoothed Dissipative Particle Dynamics (SDPD) literature. These velocity fluctuations for the fluid may be incorporated into the velocity updates for our fluid blobs to obtain a thermodynamically consistent distribution of velocities. In cases where these fluctuations are insignificant, however, these additional terms may well be dropped out as they are in a standard SPH simulation. We have applied our technique to study the rheology of two different concentrations of our model linear polymer solutions. The results show that the polymers and the fluid are coupled very well with each other, showing no lag between their velocities. Furthermore, our results show non-Newtonian shear thinning and the characteristic flattening of the Poiseuille flow profile typically observed for polymer solutions. Published by AIP Publishing. https://doi.org/10.1063/1.5006627
\end{abstract}

\section{INTRODUCTION}

Simulating the dynamics of polymers in solution has been a subject of continuous interest for almost three decades now, yet a final resolution of the problem eludes us owing to its inherent complexity. This problem poses many fundamental questions such as how do these large polymer molecules interact with the solvent fluid molecules and how do they exchange momentum under flowing conditions? Given the enormous size of the polymer molecules dissolved in a fluid made up of molecules which are orders of magnitude smaller, it is practically impossible to even simulate just tens of polymer

a)Electronic mail: v.r.ahuja@utwente.nl

b)Electronic mail: w.j.briels@utwente.nl molecules together with all the millions of fluid molecules in their vicinity if all of them are to be resolved down to the atomic level. Hence, coarse-graining is an inherent part of simulating polymers in solution.

The obvious question that arises next is what should be the level of coarse-graining, i.e., how well do we resolve the polymer molecules and the fluid? Very broadly speaking, one can categorize the simulation techniques into two major branches-particle-based simulations and Computational Fluid Dynamics (CFD) simulations using a continuum approach. Since there is a plethora of techniques under each category, we restrict the discussion here to particle-based simulation techniques, which, we might add, have the advantage of being able to link macroscopic flow behavior to microscopic interactions. Among the particle-based simulation techniques 
that have been used over the years to model the dynamics of polymers in solution, there is again an enormous variety of methods employing different levels of coarse-graining for the polymers and the fluid. There have been studies employing molecular dynamics (MD) for simulating polymers modeled as a chain of a few "monomers" connected to each other with springs in a solvent represented by a few thousand fluid particles treated as soft spheres. ${ }^{1,2}$ Hybrid models have been developed which combine two different levels of coarse-graining for the polymers and the fluid. One such hybrid model combines the MD approach for the polymer chain with the Lattice Boltzmann (LB) approach for the solvent. ${ }^{3}$ Other hybrid models combine the MD approach for the polymers with the multi-particle collision dynamics (MPCD) or Stochastic Rotation Dynamics (SRD) method for the solvent. ${ }^{4-10}$ Mesoscopic methods like Dissipative Particle Dynamics (DPD) $)^{11-13}$ have also been employed to study polymer solutions by representing the solvent with DPD particles acting as "fluid elements" and representing the polymer molecules by a chain of such DPD particles connected to each other with springs. ${ }^{14-22}$ More recently, a technique called Smoothed Dissipative Particle Dynamics (SDPD) has been developed, ${ }^{23,24}$ which is a modified version of Smoothed Particle Hydrodynamics (SPH) ${ }^{25-29}$ with added DPD-like pairwise thermal fluctuation terms. This technique has also been used for simulating polymer solutions in a similar way like DPD. ${ }^{30-32}$

Furthermore, there are other simulation techniques on a much higher level of coarse-graining based on Brownian dynamics such as Responsive Particle Dynamics (RaPiD), ${ }^{33,34}$ in which the solvent is not explicitly modeled but is rather implicitly present and the polymer molecules are represented by their centers-of-mass and interact through pair-potentials. RaPiD has been used for studying various phenomena related to polymer solutions. ${ }^{35-37}$ Recently, another technique based on Brownian dynamics with an implicit background fluid having hydrodynamic interactions was developed for modeling self-developing flow of polymer solutions in the bulk as well as in the presence of solid interfaces. ${ }^{38,39}$ As the background fluid is implicitly present at the positions of the polymer molecules, this technique is computationally efficient as it does not need separate position updates for the fluid particles. Nevertheless, the resolution of the background fluid is fixed by the concentration of the polymers and one is not free to choose an arbitrary equation of state for the fluid. To resolve these problems, it was envisaged that two different particles be used-one for modeling polymers and the other for the fluid. However, this can only be done if a proper interaction term is constructed that not only couples the motion of the polymers and the fluid but also conserves momentum so as to preserve long range hydrodynamics. This is precisely what we present in this paper. Furthermore, we have derived pairwise fluctuation terms for the fluid using the Fokker-Planck equation so that the steady state probability distributions of the positions and velocities of the fluid in a quiescent state correspond to the equilibrium distribution. We arrive essentially at the same result that has been derived using General Equation for the Non-Equilibrium Reversible-Irreversible Coupling (GENERIC) for an incompressible SDPD fluid. ${ }^{23,24}$ However, these fluctuation terms may be neglected if they do not play a major role in the case being studied, under which circumstance the fluid model reduces to standard $\mathrm{SPH}$.

\section{MODEL DEVELOPMENT}

\section{A. Equation of motion for the polymer blobs}

Consider the motion of a mesoscopic polymer blob $a$ dissolved in a solvent, alternatively referred to as the background fluid in this work, under flowing conditions. It is well known that for overdamped systems, the time scale over which the particles move to any significant extent is orders of magnitude larger than the time scale over which their velocities get completely thermalized. Therefore, these velocities may be integrated out and the positions of the particles can be updated using a first order Brownian dynamics propagator, ${ }^{40,41}$

$$
\mathrm{d} \mathbf{r}_{a}=\mathbf{v}\left(\mathbf{r}_{a}\right) \mathrm{d} t+\left(\frac{\mathbf{F}_{a}}{\xi_{a}}\right) \mathrm{d} t+k_{B} T \frac{\partial}{\partial \mathbf{r}_{a}}\left(\frac{1}{\xi_{a}}\right) \mathrm{d} t+\mathrm{d} \mathbf{W}_{a}^{r} .
$$

We would like to emphasize that $\mathbf{v}\left(\mathbf{r}_{a}\right)$, shorthand for $\mathbf{v}\left(\mathbf{r}_{a}, t\right)$, is not the velocity of the polymer blob $a$ but rather the background fluid velocity at the position of polymer blob $a$ at time $t$. $\mathbf{F}_{a}$, shorthand for $\mathbf{F}_{a}(t)$, is the driving force acting on polymer blob $a$ as a result of the interaction with other polymer blobs, in addition to any force field that may have been applied. In the remaining part of the paper, we will not include $t$ in our notation, tacitly assuming that it is implicitly present. $\xi_{a}$, shorthand for $\xi\left(\mathbf{r}_{a}\right)$, is the friction coefficient at the position of polymer blob $a$. The third term on the right-hand side of Eq. (1) is a drift term accounting for the spatial variation of the friction coefficient, which we have neglected in this study as we have assumed a constant friction coefficient for the sake of simplicity. The last term on the right-hand side of Eq. (1), i.e., $\mathbf{d} \mathbf{W}_{a}^{r}$, is a random displacement that is uncorrelated in time and has a magnitude that is calculated in accordance with the fluctuation dissipation theorem, satisfying

$$
\left\langle\mathrm{d} \mathbf{W}_{a}^{r} \mathrm{~d} \mathbf{W}_{b}^{r}\right\rangle=2 k_{B} T\left(\frac{\mathrm{d} t}{\xi_{a}}\right) \delta_{a b} \mathbf{I} .
$$

Since the background flow field is typically not known a priori, it must be calculated during the simulation in real time. We calculate this background velocity field on a moving grid of fluid blobs which serve as the node points as in a Smoothed Particle Hydrodynamics (SPH) simulation. ${ }^{25-29}$ Since the flow field is calculated only at a finite number of node points, the background fluid velocity at the position of the polymer blob $a$ in Eq. (1) must be estimated based on interpolation. To this end, we use the integral interpolant

$$
\mathbf{v}\left(\mathbf{r}_{a}\right)=\int w^{f}\left(\left|\mathbf{r}-\mathbf{r}_{a}\right|\right) \mathbf{v}(\mathbf{r}) \mathrm{d}^{3} r,
$$

where $\mathrm{d}^{3} r$ is a volume element and $w^{f}(r)$ is a normalized weight function with a cutoff $R_{c}$ satisfying for a 3-D simulation: $\int_{0}^{R_{c}} 4 \pi r^{2} w^{f}(r) \mathrm{d} r=1$. The superscript $f$ in $w^{f}(r)$ indicates that this is the weight function used for the fluid blobs. The integral in Eq. (3) can be replaced by a summation running over each of the fluid neighbors $i$ of polymer blob $a$ using the standard SPH formulation 


$$
\mathbf{v}\left(\mathbf{r}_{a}\right)=\sum_{i=1}^{N_{f}} \frac{w^{f}\left(r_{a i}\right)}{n_{i}^{f}} \mathbf{v}_{i} .
$$

Here, $N_{f}$ is the total number of fluid blobs and $r_{a i}$, shorthand for $\left|\mathbf{r}_{a}-\mathbf{r}_{i}\right|$, is the distance between polymer blob $a$ and fluid blob $i$ at time $t . n_{i}^{f}$ is the local number density of fluid blobs at the position of the fluid blob $i$ at time $t$, which is calculated by running over each of the fluid neighbors $j$ of the fluid blob $i$ using the aforementioned weight function $w^{f}(r)$,

$$
n_{i}^{f}=\sum_{j=1}^{N_{f}} w^{f}\left(r_{i j}\right) .
$$

For calculating the driving force $\mathbf{F}_{a}$ in the second term of Eq. (1), we resort to the Brownian dynamics based polymer model RaPiD, ${ }^{33}$ in which the driving force is calculated as the gradient of a two-part pair-potential,

$$
\mathbf{F}_{a}=-\frac{\partial}{\partial \mathbf{r}_{a}}\left[\Phi_{c}+\Phi_{t}\right]
$$

where $\Phi_{c}$ is the conservative potential and $\Phi_{t}$ is the transient potential, both of which shall be defined and described in Sec. III.

It is interesting to note that by rearranging Eq. (1), using Eq. (5), and assuming a constant friction coefficient $\xi$ for the sake of simplicity, one may obtain the effective frictional force $\mathbf{F}_{a}^{p f}$ acting on the polymer blob $a$ at time $t$, opposing its motion relative to the background flow field, as

$$
\mathbf{F}_{a}^{p f}=-\xi \sum_{i=1}^{N_{f}} \frac{w^{f}\left(r_{a i}\right)}{n_{i}^{f}}\left(\frac{\mathrm{d} \mathbf{r}_{a}^{*}}{\mathrm{~d} t}-\mathbf{v}_{i}\right) .
$$

Here, $\mathrm{d} \mathbf{r}_{a}^{*} / \mathrm{d} t$ is the deterministic part of the rate of change of the position of the polymer blob $a$, i.e., as calculated without the random fluctuation $\mathrm{dW}_{a}^{r}$ and $\mathbf{v}_{i}$ is the velocity of the fluid blob $i$ at time $t$. Furthermore, it can be said that it is through this frictional force that we essentially couple the motion of the polymer blobs with the motion of the background fluid. In Subsection II B, we shall refer to this frictional force to motivate the interaction force acting on the fluid blobs due to the polymer blobs.

\section{B. Equation of motion for the fluid blobs}

For calculating the background flow field, we use the incompressible Navier-Stokes equation for fluctuating hydrodynamics, ${ }^{42-45}$ augmented with an additional interaction term representing the force acting on the fluid due to the polymer,

$$
\frac{\mathrm{D} \mathbf{v}}{\mathrm{D} t}(\mathbf{r})=-\left(\frac{\boldsymbol{\nabla} P}{\rho}\right)(\mathbf{r})+\eta\left(\frac{\boldsymbol{\nabla}^{2} \mathbf{v}}{\rho}\right)(\mathbf{r})+\mathbf{g}(\mathbf{r})+\mathbf{F}^{f p}(\mathbf{r})+\mathrm{d} \mathbf{W}^{v}(\mathbf{r}) .
$$

Here, the term on the left-hand side is the material derivative of the velocity $\mathbf{v}(\mathbf{r}), \rho$ is the mass density of the fluid, $\eta$ is the viscosity of the fluid, $\nabla P(\mathbf{r})$ is the pressure gradient, $\mathbf{g}(\mathbf{r})$ is the acceleration due to body forces, $\mathbf{F}^{f p}(\mathbf{r})$ is the interaction term which represents the force on the fluid due to the polymer, and $\mathrm{d} \mathbf{W}^{v}(\mathbf{r})$ is a random fluctuation term.
Now, instead of solving the above equation on an Eulerian grid as in CFD, we discretize the above equation on a moving grid of fluid blobs as in SPH. ${ }^{25-29}$ Since the fluid blobs themselves move with the flow field, the position of a fluid blob $i$ can be updated using

$$
\mathrm{d} \mathbf{r}_{i}=\mathbf{v}_{i} \mathrm{~d} t .
$$

Here, once again we clarify that $\mathbf{r}_{i}$ is the position of fluid blob $i$ at time $t$ and $\mathbf{v}_{i}$ is the velocity of that fluid blob at that time. As a direct consequence of this, the material derivative of the velocity can be readily calculated as the rate at which the velocity of the fluid blobs changes in the Lagrangian frame of reference,

$$
\frac{\mathrm{D} \mathbf{v}}{\mathrm{D} t}\left(\mathbf{r}_{i}\right)=\frac{\mathrm{d} \mathbf{v}_{i}}{\mathrm{~d} t},
$$

and $\mathbf{g}(\mathbf{r})$ at the position of fluid blob $i$ can be discretized as $\mathbf{g}(i)$. For calculating the pressure gradient and the viscous dissipation term, standard SPH finite-difference-like forms ${ }^{29}$ have been employed,

$$
\begin{aligned}
& \left(\frac{\boldsymbol{\nabla} P}{\rho}\right)\left(\mathbf{r}_{i}\right)=\frac{1}{m} \sum_{j=1}^{N_{f}}\left(\frac{P_{i}}{\left(n_{i}^{f}\right)^{2}}+\frac{P_{j}}{\left(n_{j}^{f}\right)^{2}}\right) \frac{\mathrm{d} w^{f}}{\mathrm{~d} r}\left(r_{i j}\right) \frac{\mathbf{r}_{i j}}{r_{i j}}, \\
& \left(\frac{\boldsymbol{\nabla}^{2} \mathbf{v}}{\rho}\right)\left(\mathbf{r}_{i}\right)=\frac{1}{m} \sum_{j=1}^{N_{f}}\left(\frac{2}{n_{i}^{f} n_{j}^{f}}\right) \frac{\mathrm{d} w^{f}}{\mathrm{~d} r}\left(r_{i j}\right) \frac{\mathbf{v}_{i j}}{r_{i j}},
\end{aligned}
$$

where $\mathbf{v}_{i j}$ is the velocity of fluid blob $i$ relative to fluid blob $j$ and $m$ is the mass of the fluid blobs, which is calculated as the ratio of the mass density of the fluid $\rho$ to the average number density of the fluid blobs $\bar{n}^{f}$, i.e., $m=\rho / \bar{n}^{f}$.

$\mathbf{F}^{f p}(\mathbf{r})$ discretized at the position of the fluid blob $i$ is denoted by $\mathbf{F}_{i}^{f p}$, which represents the influence of the surrounding polymer blobs on the fluid blob $i$ at time $t$. We may now refer to the frictional force $\mathbf{F}_{a}^{p f}$ shown in Eq. (7) and construct the interaction term $\mathbf{F}_{i}^{f p}$ such that for each individual polymerfluid pair of polymer blob $a$ and fluid blob $i$, the force exerted by polymer blob $a$ on fluid blob $i$ is equal and opposite to the frictional force exerted by fluid blob $i$ on polymer blob $a$. Thus, the total force on fluid blob $i$, calculated by summing over the forces due to all the individual polymer blobs, would then be

$$
\mathbf{F}_{i}^{f p}=\xi \sum_{a=1}^{N_{p}} \frac{w^{f}\left(r_{a i}\right)}{n_{i}^{f}}\left(\frac{\mathrm{d} \mathbf{r}_{a}^{*}}{\mathrm{~d} t}-\mathbf{v}_{i}\right),
$$

where $N_{p}$ is the total number of polymer blobs. However, due to computational reasons, which shall be explained at the end of this subsection, we adopt a slightly modified version for the interaction term

$$
\mathbf{F}_{i}^{f p}=\xi \sum_{a=1}^{N_{p}} \frac{w^{f}\left(r_{a i}\right)}{n_{i}^{f}}\left(\frac{\mathrm{d} \mathbf{r}_{a}^{*}}{\mathrm{~d} t}-\mathbf{v}\left(\mathbf{r}_{a}\right)\right),
$$

where $\mathbf{v}\left(\mathbf{r}_{a}\right)$ is the velocity of the fluid interpolated at the position of polymer blob $a$ at time $t$, as defined in Subsection II A in Eq. (4). Although this means that we do not achieve pairwise momentum conservation for the polymer-fluid interactions, we still achieve momentum conservation on a local level in such a way that the total frictional force exerted on any given polymer blob $a$ by the fluid is distributed back in its entirety to the fluid 
blobs in its local vicinity, the proof of which has been provided in Appendix A. We can further simplify $\mathbf{F}_{i}^{f p}$ using Eqs. (1) and (14) to arrive at

$$
\mathbf{F}_{i}^{f p}=\sum_{a=1}^{N_{p}} \frac{w^{f}\left(r_{a i}\right)}{n_{i}^{f}} \mathbf{F}_{a}
$$

We must emphasize that it is through this term that the fluid blobs experience the influence of the polymer blobs. Thus, the two interaction terms $\mathbf{F}^{p f}$ and $\mathbf{F}^{f p}$ essentially make this model a two-way coupling scheme. We might add that this strategy of redistributing the force is reminiscent of a two-way coupling model that was developed for gas-fluidized beds. ${ }^{46}$

Combining the results from Eqs. (8), (10)-(12), and (15) and discretizing the fluctuation term $\mathrm{dW}^{v}(\mathbf{r})$ at the position of the fluid blob $i$ as a pairwise sum of random terms denoted by $\mathrm{dW}_{i j}^{v}$, we arrive at the following equation that we have used in our simulations to update the velocities of the fluid blobs:

$$
\begin{aligned}
\mathrm{d} \mathbf{v}_{i}= & -\frac{\mathrm{d} t}{m}\left[\sum_{j=1}^{N_{f}}\left(\frac{P_{i}}{\left(n_{i}^{f}\right)^{2}}+\frac{P_{j}}{\left(n_{j}^{f}\right)^{2}}\right) \frac{\mathrm{d} w^{f}}{\mathrm{~d} r}\left(r_{i j}\right) \frac{\mathbf{r}_{i j}}{r_{i j}}+\sum_{j=1}^{N_{f}} f_{i j} \mathbf{v}_{i j}\right] \\
& +\mathbf{g}_{i} \mathrm{~d} t+\frac{\mathrm{d} t}{m}\left[\sum_{a=1}^{N_{p}} \frac{w^{f}\left(r_{a i}\right)}{n_{i}^{f}} \mathbf{F}_{a}\right]+\sum_{j=1}^{N_{f}} \mathrm{~d} \mathbf{W}_{i j}^{v},
\end{aligned}
$$

where $f_{i j}$, shorthand for $f\left(r_{i j}\right)$, is a symmetric function defined as follows:

$$
f\left(r_{i j}\right)= \begin{cases}-\left(\frac{2 \eta}{n_{i}^{f} n_{j}^{f}}\right) \frac{1}{r_{i j}} \frac{\mathrm{d} w^{f}}{\mathrm{~d} r}\left(r_{i j}\right) & \text { for } r_{i j} \leq R_{c}, \\ 0 & \text { for } r_{i j} \geq R_{c} .\end{cases}
$$

The pairwise velocity fluctuation terms are uncorrelated in time and have been calculated in an anti-symmetric manner such that $\mathrm{d}_{\mathbf{W}}^{v}=-\mathrm{d} \mathbf{W}_{j i}^{v}$ so that the velocity updates are momentum conserving. The properties of these momentum fluctuations $\mathrm{d} \mathbf{W}_{i j}$ have been calculated in a way that the steady state probability distribution of the positions and velocities of the fluid in a quiescent state yields the expected equilibrium distribution. From a detailed derivation shown in Appendix B, we have

$$
\begin{aligned}
\left\langle\mathrm{d} \mathbf{W}_{i j}^{v} \mathrm{~d} \mathbf{W}_{i j}^{v}\right\rangle & =\left(\frac{2 k_{B} T}{m}\right)\left(\frac{\mathrm{d} t}{m}\right) f_{i j} \mathbf{I}, \\
\left\langle\mathrm{d} \mathbf{W}_{i k}^{v} \mathrm{~d} \mathbf{W}_{j l}^{v}\right\rangle & =\mathbf{0} \quad(i k \neq j l \wedge i k \neq l j) .
\end{aligned}
$$

Notice that if instead of Eq. (14) a pairwise anti-symmetric interaction term as shown in Eq. (13) would have been used, then we would have ended up with an additional term proportional to the product of the time step $\mathrm{d} t$ and the friction coefficient $\xi$ in the equation of motion for the fluid blobs. This would deprive us of the whole advantage of using Brownian dynamics for the polymers which allows us to use a larger time step for overdamped systems where the friction coefficient $\xi$ is large. This is the reason for not using Eq. (13) as the interaction term for the fluid blobs.

It is also interesting to note that if we would not have neglected the spatial variation of the friction coefficient in Eq. (1), then we would have had an additional term proportional to $\frac{\partial}{\partial \mathbf{r}_{a}} \ln \left(\xi_{a}\right)$ in the equation of motion for the fluid blobs.
However, since this term grows only weakly with increasing $\xi_{a}$, it would not affect our proposed technique significantly.

\section{TEST SYSTEM AND PARAMETERS}

\section{A. The conservative potential}

We have tested our technique with a model linear polymer solution at different concentrations. We use the Flory-Huggins potential, which has been used in the literature for describing the conservative part of the interaction potential between the linear polymers, ${ }^{35-37}$ defined as follows:

$$
\Phi_{c}=p k_{B} T \sum_{a=1}^{N_{p}}\left[\left(\frac{1-\phi_{a}}{\phi_{a}}\right) \ln \left(1-\phi_{a}\right)-\chi \phi_{a}\right] .
$$

Here, $N_{p}$ is the total number of polymer blobs in solution as mentioned before, $\Phi_{c}$ is the conservative potential, $p$ is the number of Kuhn segments in a polymer blob, $\chi$ is the solvent interaction parameter, and $\phi_{a}$ is the local volume fraction of polymer blobs in the neighborhood of polymer blob $a$ at time $t$ calculated as

$$
\phi_{a}=\frac{n_{a}^{p}}{n_{\max }^{p}} .
$$

Here, $n_{\text {max }}^{p}$ is the maximum number density of polymers that the system is allowed to reach, i.e., the melt density, and $n_{a}^{p}$ is the local number density of polymer blobs at the position of polymer blob $a$ calculated as

$$
n_{a}^{p}=\sum_{b=1}^{N_{p}} w^{p}\left(r_{a b}\right)
$$

where $r_{a b}$ is the distance between polymers $a$ and $b$ at time $t$ and $w^{p}(r)$ is a normalized weight function with a cutoff $r_{c}$ satisfying for a 3-D simulation: $\int_{0}^{r_{c}} 4 \pi r^{2} w^{p}(r) \mathrm{d} r=1$. The superscript $p$ in $w^{p}(r)$ indicates that this is the weight function used for the polymer blobs.

\section{B. The transient potential}

We have used the RaPiD technique to incorporate memory effects into the simulation model through a transient potential, which depends on the history of the interacting polymers by keeping track of dynamic variables, given by ${ }^{33,34}$

$$
\Phi_{t}=\frac{1}{2} \alpha \sum_{a, b=1}^{N_{p}}\left(\lambda_{a b}-\lambda_{a b}^{e q}\right)^{2} .
$$

Here, $\Phi_{t}$ is the transient potential, $\alpha$ is a parameter associated with the strength of the interactions or, in other words, the penalty for the deviation of the dynamic variable $\lambda_{a b}$ from its equilibrium value $\lambda_{a b}^{e q}$. The variable $\lambda_{a b}$, shorthand for $\lambda_{a b}(t)$, is a dimensionless dynamic variable representing the degree of intermixing of the polymers $a$ and $b$, which evolves over time based on the following first order stochastic differential equation:

$$
\mathrm{d} \lambda_{a b}=-\left(\lambda_{a b}-\lambda_{a b}^{e q}\right) \frac{\mathrm{d} t}{\tau}+\mathrm{d} \mathbf{W}_{a b}^{\lambda},
$$

where $\tau$ is the relaxation time and $\mathbf{W}_{a b}^{\lambda}$ is a Wiener process with time-uncorrelated increments satisfying 


$$
\left\langle\mathrm{d} \mathbf{W}_{a b}^{\lambda} \mathrm{d} \mathbf{W}_{a b}^{\lambda}\right\rangle=\left(\frac{2 k_{B} T}{\alpha}\right)\left(\frac{\mathrm{d} t}{\tau}\right) \mathbf{I} .
$$

The variable $\lambda_{a b}^{e q}$, shorthand for $\lambda^{e q}\left(r_{a b}\right)$, is the equilibrium value of the variable $\lambda_{a b}$ for the distance $r_{a b}$, shorthand for $r_{a b}(t)$, between polymers $a$ and $b$ at time $t$. For the definition of $\lambda_{a b}^{e q}$, we use here the following form that has been used in the literature: ${ }^{35-37}$

$$
\lambda^{e q}\left(r_{a b}\right)= \begin{cases}\left(1-\frac{r_{a b}}{r_{c}}\right)^{2} & \text { for } r_{a b} \leq r_{c} \\ 0 & \text { for } r_{a b} \geq r_{c}\end{cases}
$$

\section{The equation of state}

For the fluid, we use the commonly used pseudoincompressible equation of state, which is essentially a modified version of the Tait equation of state with the exponent chosen as 7 so that it works well for water, ${ }^{47,48}$

$$
P_{i}=P_{0}\left[\left(\frac{n_{i}^{f}}{\bar{n}^{f}}\right)^{7}-1\right]
$$

where $P_{0}$ is chosen such that the velocity of sound in the simulation is sufficiently large in order that the density fluctuations are sufficiently small, which results in a fluid that resembles an incompressible fluid.

\section{Definition of weight functions and system parameters}

For the polymer blobs, we have used a normalized weight function that has been used earlier in the literature, where the polymer model RaPiD has been employed. ${ }^{35-37}$ It is a monotonically decreasing function with derivatives up to the first order continuous to prevent any discontinuities in the forces which are related to the first derivative of the weight function. Furthermore, it has a non-zero derivative at the origin in order to have a net repulsive force at zero-distance to prevent clustering. It is given by $w^{p}(r)$,

$$
w^{p}(r)= \begin{cases}\frac{15}{2 \pi\left(r_{c}^{5}-\sigma^{5}\right)}\left(r_{c}-\sigma\right)\left(r_{c}+\sigma-2 r\right) & \text { for } r \leq \sigma, \\ \frac{15}{2 \pi\left(r_{c}^{5}-\sigma^{5}\right)}\left(r-r_{c}\right)^{2} & \text { for } \sigma \leq r \leq r_{c}, \\ 0 & \text { for } r \geq r_{c},\end{cases}
$$

where the cutoff $r_{c}$ is chosen as $2.5 \sigma$. It can be easily checked that the weight function $w^{p}(r)$ is normalized in 3-dimensions within this cutoff radius $r_{c}$ such that $\int_{0}^{r_{c}} 4 \pi r^{2} w^{p}(r) d r=1$.

For the fluid blobs, we have chosen the normalized $M_{4}$ kernel commonly used in $\mathrm{SPH}^{29}$ as the weight function. It is a cubic spline (piecewise continuous polynomial of degree 3 ) having derivatives up to the second order continuous and a cutoff of $R_{c}=2 h$. It is given by $w^{f}(r)$,

$$
w^{f}(r)= \begin{cases}\frac{1}{4 \pi h^{3}}\left[\left(2-\frac{r}{h}\right)^{3}-4\left(1-\frac{r}{h}\right)^{3}\right] & \text { for } r \leq h, \\ \frac{1}{4 \pi h^{3}}\left(2-\frac{r}{h}\right)^{3} & \text { for } h \leq r \leq 2 h, \\ 0 & \text { for } r \geq 2 h,\end{cases}
$$

where $h$ is what is commonly referred to as the support of the weight function. Again, it can be easily checked that the weight function $w^{f}(r)$ is normalized in 3-dimensions within the cutoff radius such that $\int_{0}^{R_{c}} 4 \pi r^{2} w^{f}(r) d r=1$.

We have chosen $h=2 \sigma$ for our simulations such that the cutoff radius $R_{c}$ for the fluid blobs is larger than the cutoff radius $r_{c}$ chosen for the polymer blobs because the weight function $w^{f}(r)$ must be able to accurately estimate the secondorder derivatives occurring in the equation of motion for the fluid blobs. Following the same logic, it immediately follows that for the polymer-fluid interactions, as we do not need to calculate any gradients, we can use the weight function $w^{p}(r)$ with a smaller cutoff $r_{c}$ instead of $w^{f}(r)$ with a larger cutoff $R_{c}$ for computational efficiency. The values of the other system parameters have been summarized in Table I.

The physical properties of the fluid, i.e., the density and viscosity, have been chosen to be consistent with those of water. The value of $P_{0}$ has been chosen high enough to ensure small density fluctuations and a large enough velocity of sound $c_{s}$ in the simulation, which can be calculated as

$$
c_{s}=\sqrt{\frac{\partial P}{\partial \rho}}=\sqrt{\frac{7 P_{0}}{\rho}} .
$$

For the value of $P_{0}$ that we have used, the velocity of sound in the simulation calculated using the above equation is about $0.03 \mathrm{~m} / \mathrm{s}$. We have ensured that the velocities that we encounter in the simulations that we have performed in this study are much smaller than this velocity of sound in our simulation.

The length scale $\sigma$ has been purposefully chosen to be large for computational reasons so as to be able to use a large time step as the main intention here is to show the two-way coupling between the polymers and the fluid through our interaction term. Although for the fluid blobs this length scale is reasonable as it is motivated phenomenologically using SPH, for the polymer blobs which are motivated using a polymer model at the mesoscopic level, it is much larger than the usual molecular level. However, if one were to use polymers which are much smaller, then one must also use smaller fluid blobs because otherwise the velocity gradients of the fluid will not be

TABLE I. Summary of system parameters.

\begin{tabular}{lccc}
\hline \hline System parameter & Symbol & Value & Unit \\
\hline Length scale & $\sigma$ & 5.0 & $\mu \mathrm{m}$ \\
Time step & $\mathrm{d} t$ & 1.0 & $\mu \mathrm{s}$ \\
Temperature & $T$ & 300 & $\mathrm{~K}$ \\
Density of fluid & $\rho$ & 1000 & $\mathrm{~kg} / \mathrm{m}^{3}$ \\
Viscosity of fluid & $\eta$ & 1.0 & $\mathrm{mPa} \mathrm{s}$ \\
Resolution of fluid & $\bar{n}^{f}$ & $1.9099 \times 10^{15}$ & $\mathrm{Particles}\left(\mathrm{m}^{3}\right)$ \\
Pressure coefficient & $P_{0}$ & 0.13 & $\mathrm{~Pa}$ \\
Friction coefficient & $\xi$ & $1.0 \times 10^{-7}$ & $\mathrm{~kg} / \mathrm{s}$ \\
Strength of polymer interactions & $\alpha$ & 500 & $k_{B} T$ \\
Relaxation time & $\tau$ & 1.0 & $\mathrm{~s}$ \\
Number of Kuhn segments & $\mathrm{p}$ & 300000 & $\ldots$ \\
Maximum number density & $n_{\max }$ & $1.0 \times 10^{4}$ & $\mathrm{C}^{*}$ \\
of polymers & & & \\
Flory-Huggins interaction & $\chi$ & 0.5 & $\ldots$ \\
parameter & & & \\
\hline \hline
\end{tabular}


accurately experienced by the polymer blobs thereby adversely affecting the hydrodynamic coupling. This would lead to a smaller time step and, on the other hand, if larger fluid blobs are used, then the number of polymers required becomes very large and we run into computational problems again as will be described in more detail in Sec. V. Furthermore, due to the large size of the polymer blobs, the concentration $\mathrm{C}^{*}$, which is calculated as the concentration at which there is on average 1 particle in a volume of a sphere of radius $\sigma$, is rather small because this sphere is quite large. Hence, we have chosen large values for parameters like the effective Kuhn segments $p$ of our polymer blob, the strength of inter-polymer interactions $\alpha$, and the maximum number density of polymers $n_{\max }^{p}$, which is a Flory-Huggins parameter that typically corresponds to the melt density. For the Flory-Huggins interaction parameter $\chi$, we have chosen the limiting value of 0.5 , above which phase separation would occur, which is not what we aim for in this study. Nevertheless, depending on the system being modeled, i.e., depending on whether the solvent is a good solvent or a bad solvent for the polymer, the value of the Flory-Huggins parameter $\chi$ may be selected accordingly and our method of coupling the polymer and the fluid would still work. We want to emphasize that the term polymer blob in this paper refers to a whole polymer molecule or even a collection of a few polymer molecules in a single blob, which is slightly different from the usual usage of the word blob referring to a collection of a few monomers.

\section{RESULTS AND DISCUSSION}

\section{A. Pure fluid simulations}

In this subsection, we present simulations of the pure fluid, i.e., in the absence of the polymers. Since the equation of motion for the fluid is based on the Navier-Stokes equation for an incompressible Newtonian fluid, we expect to see the Newtonian behavior in the dynamic properties of the pure fluid.

\section{Equilibrium simulation}

Consider a fluid blob in a sea of fluid blobs of an incompressible Newtonian fluid in a quiescent state. At equilibrium, although the mean velocity of the fluid blob is zero, at any given instant it has a finite instantaneous velocity due to thermal fluctuations. Although these instantaneous velocities are random both in magnitude and direction, we can still say something about the distribution of these velocities and the rate at which these velocities dissipate from the kinetic and hydrodynamic theories, respectively. It is well known that at thermal equilibrium, the velocities must obey the Maxwell-Boltzmann distribution, and therefore for any given component of velocity $v_{0}$, we have

$$
\left\langle v_{0}^{2}\right\rangle=k_{B} T / m,
$$

where $k_{B}$ is the Boltzmann constant, $T$ is the temperature of the system, and $m$ is the mass of the fluid blob. Furthermore, from hydrodynamic theory for Newtonian liquids, it is known that at short times and large wavelengths, the transverse current correlation function decays exponentially over time and the time constant is related to the kinematic shear viscosity

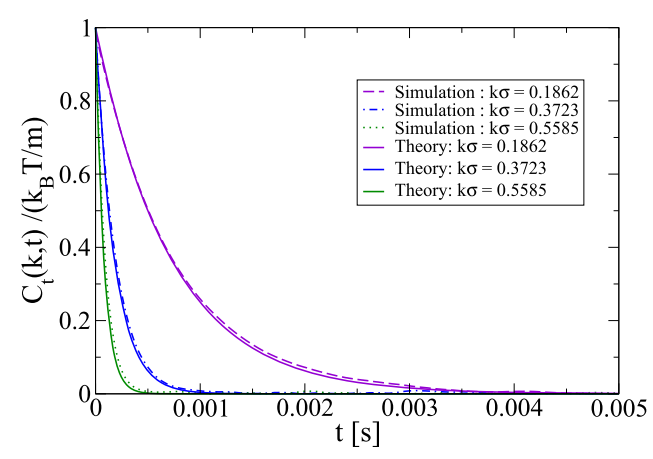

FIG. 1. Normalized transverse current correlation function for the pure fluid at equilibrium for three different wave vectors.

of the system. So, we have calculated the transverse current correlation function $C_{t}(k, t)$ for velocities in the y-direction and wave vectors in the $\mathrm{x}$-direction $\left(\mathbf{k}_{x}=k \hat{\mathbf{e}}_{x}\right)$,

$$
\left.C_{t}(k, t)=\frac{1}{N_{f}} \tilde{j_{y}}\left(\mathbf{k}_{x}, t\right) \tilde{j}_{y}^{*}\left(\mathbf{k}_{x}, 0\right)\right\rangle,
$$

where $N_{f}$ is the total number of fluid blobs and the transverse current $\tilde{j}_{y}\left(\mathbf{k}_{\mathbf{x}}, t\right)$ is defined as follows:

$$
\tilde{j}_{y}\left(\mathbf{k}_{\mathbf{x}}, t\right)=\hat{\mathbf{e}}_{y} \cdot \sum_{j=1}^{N_{f}} \mathbf{v}_{j}(t) e^{-i \mathbf{k}_{x} \cdot \mathbf{r}_{j}(t)},
$$

where $\mathbf{r}_{j}(t)$ is the position of fluid blob $j$ at time $t$ and $\mathbf{v}_{j}(t)$ is its instantaneous velocity at that time. From hydrodynamic theory, it is known that at short times and small $k$-values, the transverse current correlation function $C_{t}(k, t)$, defined above,

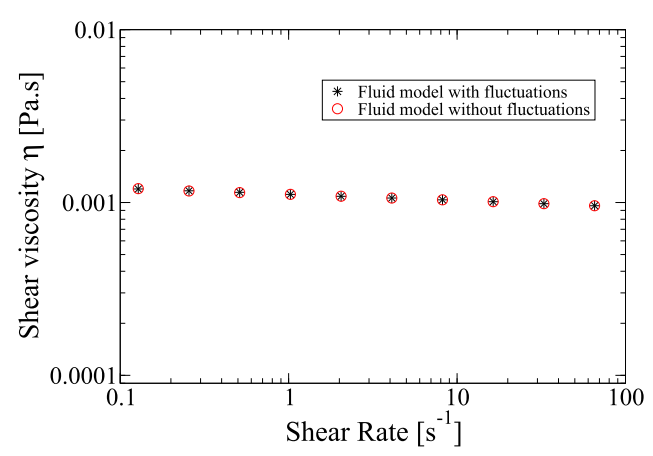

FIG. 2. Flow curve for the pure fluid model with and without thermal fluctuations.

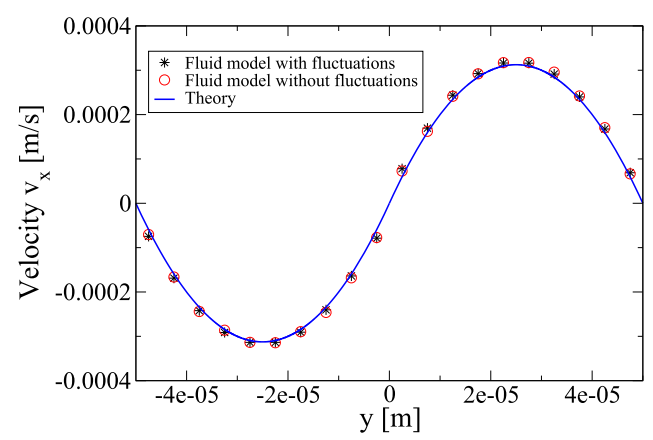

FIG. 3. Reverse Poiseuille flow profile-comparison of simulation results with theory. 


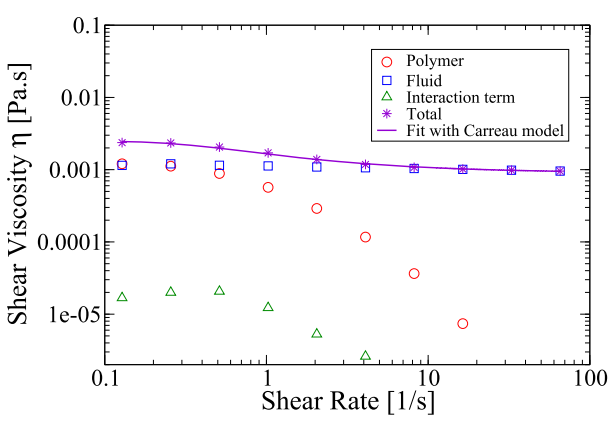

(a)

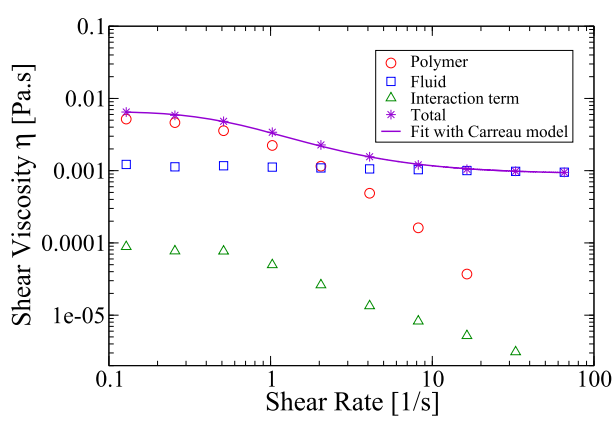

(b)
FIG. 4. Flow curves for different concentrations of polymer solution showing split-up of the various contributions to the shear viscosity. (a) Concentration $C_{1}$ $=2.5 \mathrm{C}^{*}$. (b) Concentration $C_{2}=5 \mathrm{C}^{*}$. decays as follows: ${ }^{49}$

$$
C_{t}(k, t)=\left\langle v_{0}^{2}\right\rangle e^{-v k^{2} t}
$$

where $v$ is the kinematic shear viscosity of the fluid and $v_{0}$ is the initial value of the $y$-component of the velocity. Combining Eqs. (31) and (34), we have

$$
C_{t}(k, t)=\left(\frac{k_{B} T}{m}\right) e^{-v k^{2} t} .
$$

Since we need small $k$-values, i.e., large wavelengths in the $\mathrm{x}$-direction, we have chosen a simulation box with a length $L_{x}=33.75 \sigma$ in the x-direction and $10 \sigma$ in the other twodirections and three different $\mathrm{k}$ values of $2 \pi / L_{x}, 4 \pi / L_{x}$, and $6 \pi / L_{x}$, i.e., $0.1862 \sigma^{-1}, 0.3723 \sigma^{-1}$, and $0.5585 \sigma^{-1}$, respectively. As we can see from Fig. 1, there is a good match between the simulation results and the theoretical curves, particularly for the smallest $k$-value at small times, as was to be expected, indicating that the model is hydrodynamically consistent. Furthermore, it ascertains that the velocity fluctuation terms that we have derived based on the Fokker-Planck equation in Appendix B do indeed maintain the correct kinetic temperature and that the system has the right kinematic shear viscosity.

\section{Shear flow simulations}

We have performed shear flow simulations with our fluid model for a number of different shear rates. Essentially, in every simulation, we have maintained a constant shear rate across the box using the Lees-Edwards technique ${ }^{50}$ and measured the average stress in the box over a period of time. From this, we have calculated how the viscosity varies with the shear rate as shown in Fig. 2.

We have performed these shear flow simulations using our fluid model with thermal fluctuations as well as by turning off the fluctuations. As we can see from Fig. 2, the viscosity is close to the viscosity of water $(1 \mathrm{mPa} s)$ for the range of shear rates that we have considered, given the parameters and resolution of the fluid that we have used. Although the viscosity does vary by about $15 \%$, this is to be expected given the finite resolution of the fluid typically used in SPH simulations. More importantly, it is consistent with the viscosity that can be interpreted from the transverse current correlation function shown in Subsection IV A 1.

\section{Reverse Poiseuille flow simulation}

In this subsection, we present simulations where the shear rate varies across the box in the $y$-direction as opposed to the simulations shown in Subsection IV A 2, where there was a homogeneous shear flow. We essentially achieve this by applying a constant gravitational force field in the $\mathrm{x}$-direction in the top half of the box and an equal and opposite field in the lower half of the box which leads to the development of a Poiseuille flow profile in both halves of the box. This method is what is commonly referred to as the "Reverse Poiseuille flow" in the literature. ${ }^{51}$ We have performed this simulation for the fluid model with and without fluctuations and compared the results with the analytical profile that can be calculated from a theory based on the Navier-Stokes equation for a Newtonian fluid. As can be seen from Fig. 3, there is a good match between simulations and theory.

Furthermore, it can be seen from Figs. 2 and 3 that the fluctuation terms do not affect the fluid viscosity. Hence, for the sake of computational efficiency, we do not include the thermal fluctuations in Sec. IV B where we have polymer blobs as well.

\section{B. Polymer solution simulations}

For this study, we have considered an aqueous solution of a model polymer system, where the polymer blobs interact with each other through the conservative and transient interactions formulated by the RaPiD model mentioned in Subsections III A and III B. The model for the aqueous solvent is the same as the one we have used in Subsection IV A but without thermal fluctuations. The polymers and the fluid blobs of the aqueous solvent interact with each other through the interaction terms that we have defined in Sec. II.

\section{Shear flow simulations}

We have performed shear flow simulations for our model linear polymer solutions of two different concentrations $C_{1}=2.5 \mathrm{C}^{*}$ and $C_{2}=5 \mathrm{C}^{*}$ and the results have been plotted in Fig. 4. The different contributions to the shear viscosity due to the polymers, the fluid, and the interaction term have

TABLE II. Parameters for the Carreau model fit.

\begin{tabular}{lcccc}
\hline \hline Fit parameter & Symbol & $C_{1}$ & $C_{2}$ & Unit \\
\hline Zero shear rate viscosity & $\eta_{0}$ & 2.5260 & 6.6688 & $\mathrm{mPa} \mathrm{s}$ \\
Infinite shear rate viscosity & $\eta_{\infty}$ & 0.9 & 0.9 & $\mathrm{mPa} \mathrm{s}$ \\
Fit parameter 1 & $\lambda$ & 3.0504 & 2.1408 & $\mathrm{~s}$ \\
Fit parameter 2 & $n$ & 0.3441 & 0.0008 & $\ldots$ \\
\hline \hline
\end{tabular}




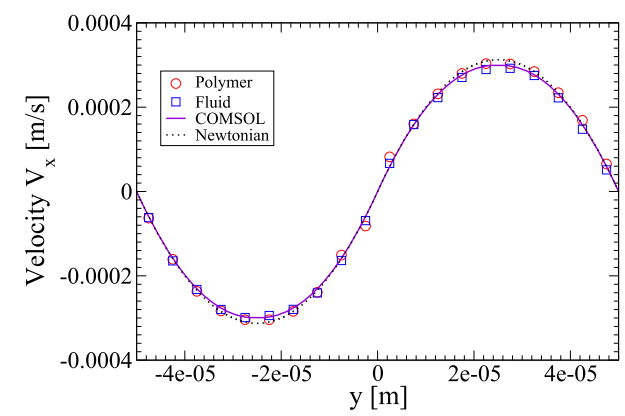

(a)

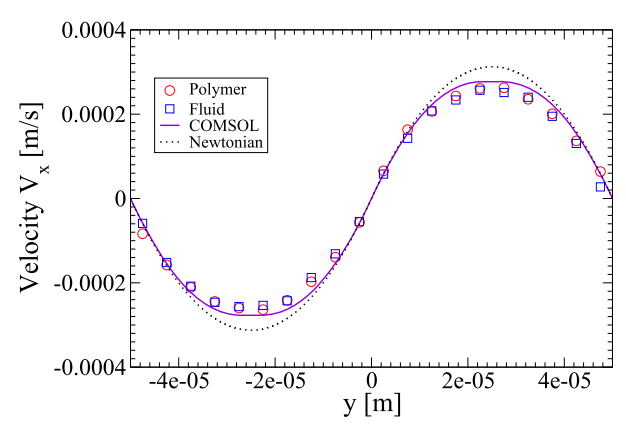

(b)
FIG. 5. Reverse Poiseuille flow profiles for different concentrations of polymer solutions showing the comparison of the polymer and fluid velocity profiles in the simulation with the CFD profile generated using COMSOL and the analytical Newtonian profile. (a) Concentration $C_{1}$ $=2.5 \mathrm{C}^{*}$. (b) Concentration $C_{2}=5 \mathrm{C}^{*}$. been shown. We have fitted the total shear viscosity flow curve with the Carreau model, which is commonly used for polymer solutions, given by ${ }^{52}$

$$
\frac{\eta-\eta_{\infty}}{\eta_{0}-\eta_{\infty}}=\left[1+(\lambda \dot{\gamma})^{2}\right]^{\frac{n-1}{2}}
$$

where $\eta_{0}$ is the zero shear rate viscosity, $\eta_{\infty}$ is the infinite shear rate viscosity, $\lambda$ is a parameter with units of time, and $n$ is a dimensionless parameter.

The fit parameters of the Carreau model that best represents the total viscosity for the two different concentrations of polymer solutions that we have studied are presented in Table II. As can be seen from Fig. 4, the solution is non-Newtonian in nature and the effect is clearly significant at higher concentrations. This shear thinning is mainly due to the transient interactions between the polymer blobs which are weaker at higher shear rates.

\section{Reverse Poiseuille flow simulations}

We have also performed reverse Poiseuille flow simulations for our model linear polymer solutions at the two concentrations $C_{1}=2.5 \mathrm{C}^{*}$ and $C_{2}=5 \mathrm{C}^{*}$. We have compared the results from our simulations with $\mathrm{CFD}$ simulations performed with the commercial package COMSOL ${ }^{53}$ to which we fed as input the Carreau model flow curve that we have shown in Subsection IV B 1. The results have been plotted in Fig. 5.

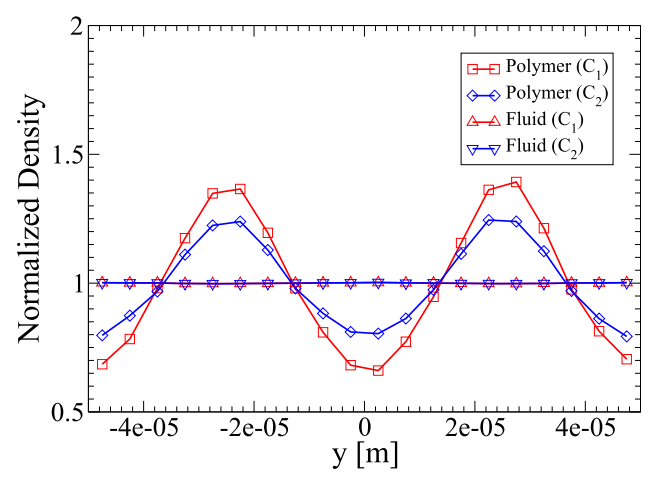

FIG. 6. Normalized density profile for fluid and polymer blobs for different concentrations of polymer solutions, viz., $C_{1}=2.5 \mathrm{C}^{*}$ and $C_{2}=5 \mathrm{C}^{*}$. Note that the density of the blobs has been normalized with the mean density of those blobs calculated with the respective weight function in that particular simulation.
As can be seen from Fig. 5, there is no lag between the polymer blobs and the fluid blobs as their velocity profiles coincide with each other, which indicates that our interaction term couples the fluid and the polymers well. Furthermore, our model for the non-Newtonian polymer solutions is able to reproduce the characteristic flattening of the velocity profile, as is evident by comparison with the parabolic Newtonian profile particularly for higher concentrations.

Our simulations also show the cross-stream migration phenomenon observed for polymers in solution. ${ }^{54}$ As can be seen from Fig. 6, the concentration of water blobs remains constant along the y-axis, i.e., along the gradient direction. The polymer concentration, however, has minima at the regions of maximum shear and maxima at the regions of zero shear. Notice that the densities of the blobs have been normalized with their respective mean density in that particular solution. Therefore, the maximum concentration of the polymers in the zero-shear region in absolute terms is higher for the polymer solution with concentration $C_{2}$ as compared to that for the polymer solution with concentration $C_{1}$ although it might seem otherwise on a first glance by looking at the normalized densities plotted in Fig. 6.

\section{CONCLUSION AND SCOPE FOR FURTHER RESEARCH}

We have presented a novel technique, hereafter referred to as Hydrodynamically Coupled Brownian Dynamics (HCBD), which is essentially a momentum conserving two-way coupling algorithm that couples the motion of polymers moving as per Brownian dynamics with the motion of the fluid which is calculated using the SPH methodology based on the numerical solution of the Navier-Stokes equation discretized on a moving grid of fluid particles. We have calculated fluctuation terms for the update of the fluid velocities based on the FokkerPlanck equation, which effectively renders our fluid model into a discretized version of fluctuating hydrodynamics. ${ }^{42-45}$ We essentially arrive at the result for an incompressible SDPD fluid derived using the GENERIC approach. ${ }^{23,24}$ Nevertheless, for computational efficiency, these fluctuating terms may be dropped out and then the pure fluid model, i.e., in the absence of the polymers, reduces to the standard SPH formulation. The main achievement of this study is the construction of the interaction term between the Brownian polymer model and the SPH fluid model. This interaction term couples the motion of the polymers to that of the fluid without any lag and also 
conserves momentum at a local level, preserving long range hydrodynamics.

The merits of this HCBD model as a technique for modeling self-developing flow of polymer solutions can be better understood if it is compared with a standard Brownian dynamics simulation. In a standard Brownian dynamics simulation, if the friction is to be applied with respect to a non-static background fluid, then an additional term describing the motion of the background fluid must be added to the Brownian dynamics propagator. However, typically the background fluid velocity is not known a priori particularly if the fluid is flowing through a complex geometry. Even if independent simulations are carried out to determine the flow profile of the fluid, which then is added to the motion of the Brownian propagator for the polymers, there is still a lag observed between the polymers and the fluid. More importantly, the fluid velocity profile experiences no influence from the motion of the polymers, thereby making it a one-way coupling scheme. In our HCBD scheme, on the other hand, the fluid and the polymer are coupled with each other through the frictional interaction term that does not lead to any lag and makes it a two-way coupling scheme. This enables us to model the flow of the polymer solution as it develops in space and time. Furthermore, since our fluid model is based on SPH, it is easy to incorporate boundary conditions such as the no-slip boundary condition at the solid interfaces in a similar way to what we have shown in an earlier work. $^{39}$

One of the consequences of modeling the polymers at a mesoscopic scale while describing the fluid using a phenomenological approach is that if one wishes to accurately model the scale of the polymers, then one must also use fluid blobs that are not orders of magnitude larger, otherwise velocity gradients of the fluid are no longer captured accurately. This can affect the hydrodynamics of the system adversely. Even so, if larger fluid blobs are used so that a larger time step may be used, still the ratio between the number of polymers and the number of fluid blobs becomes large, making the simulation computationally intensive. However, the computation time may be substantially reduced by massive parallelization, which in principle, our propagators allow for and it would still be faster than an MD simulation of course as we exploit the advantage of coarse-graining.

It is also interesting to note that if one imagines a hypothetical situation in which a fluid blob is centered at the position of the center-of-mass of each polymer blob, not necessarily the same fluid blob at all times, then the position update for the polymer blob and the velocity update for the fluid blob moreor-less reduce to that of the model described in our recently published work, in which the fluid velocities are calculated at the positions of the centers-of-mass of the polymers and hence no interpolation is required. ${ }^{38,39}$ In that model, it was proposed that the force on the polymers be immediately transmitted to the fluid, the rationality of which, in a way, has been justified through this paper, where we indeed arrive at it through the construction of the frictional force between the polymers and the fluid which couples the two together. Having said that, the model presented in this present paper has several advantages over the earlier model such as being able to independently choose an equation of state for the fluid through the pressure term and being able to independently decide the resolution of fluid irrespective of the concentration of the polymers. Moreover, although it might seem that the earlier model is more efficient than the present model because the former model did not have to move the fluid around separately, yet the latter model can actually turn out to be computationally more efficient in certain cases. This is because we can reduce the number of fluid particles in the system by choosing a resolution independent of the concentration of the polymers limited only by the dimensions of the complex geometry through which it flows.

Another important point is that the methodology presented in this paper allows one to use any polymer model of one's choice and combine it with the SPH fluid model. The polymer model that we have used in this study, i.e., RaPiD, has the limitation that it does not effectively capture all the internal dynamics of the polymer molecules but rather mainly relies on inter-polymer interactions to produce a non-Newtonian response like the shear-thinning behavior. However, as mentioned before, using our technique of coupling presented in this paper, i.e., HCBD, one can couple the SPH-based fluid model to any other polymer model. For instance, one could use an extension of the RaPiD model, ${ }^{37}$ where the polymer is not just represented by its center-of-mass alone but by a finitely extensible non-linear elastic dumbbell that interacts with other such dumbbells using the usual RaPiD potentials. This would be one way of incorporating some internal dynamics into the polymer model thereby more accurately modeling viscoelastic polymeric liquids without affecting the computational efficiency too much. This provides scope for further research on this topic.

\section{ACKNOWLEDGMENTS}

This work is part of the Industrial Partnership Programme (IPP) "Computational sciences for energy research" of the Foundation for Fundamental Research on Matter (FOM), which is part of the Netherlands Organisation for Scientific Research (NWO). This research programme is co-financed by Shell Global Solutions International B.V. J. van der Gucht acknowledges the European Research Council for financial support (ERC Consolidator grant Softbreak).

\section{APPENDIX A: PROOF OF LOCAL MOMENTUM CONSERVATION FOR THE INTERACTION BETWEEN POLYMER AND FLUID BLOBS}

Using Eqs. (4) and (14), we obtain the total force due to polymer blob $a$ on the fluid in its local vicinity, denoted by $\mathbf{F}_{a}^{f p}$, as follows:

$$
\mathbf{F}_{a}^{f p}=\xi \sum_{i=1}^{N_{f}} \frac{w^{f}\left(r_{a i}\right)}{n_{i}^{f}}\left(\frac{\mathrm{d} \mathbf{r}_{a}^{*}}{\mathrm{~d} t}-\sum_{j=1}^{N_{f}} \frac{w^{f}\left(r_{a j}\right)}{n_{j}^{f}} \mathbf{v}_{j}\right) .
$$

Simplifying the first term using Eq. (5) and rewriting the second term as two terms, one where $j=i$ and one where $j \neq i$, we obtain 


$$
\begin{aligned}
\mathbf{F}_{a}^{f p}= & \xi\left[\frac{\mathrm{d} \mathbf{r}_{a}^{*}}{\mathrm{~d} t}-\sum_{i=1}^{N_{f}}\left(\frac{w^{f}\left(r_{a i}\right)}{n_{i}^{f}}\right)^{2} \mathbf{v}_{i}\right. \\
& \left.-\left(\sum_{i=1}^{N_{f}} \frac{w^{f}\left(r_{a i}\right)}{n_{i}^{f}} \sum_{j \neq i}^{N_{f}} \frac{w^{f}\left(r_{a j}\right)}{n_{j}^{f}} \mathbf{v}_{j}\right)\right] .
\end{aligned}
$$

Rewriting the last term and simplifying, we get

$$
\begin{aligned}
\mathbf{F}_{a}^{f p}= & \xi\left[\frac{\mathrm{d} \mathbf{r}_{a}^{*}}{\mathrm{~d} t}-\sum_{i=1}^{N_{f}}\left(\frac{w^{f}\left(r_{a i}\right)}{n_{i}^{f}}\right)^{2} \mathbf{v}_{i}\right. \\
& \left.-\left(\sum_{j=1}^{N_{f}} \frac{w^{f}\left(r_{a j}\right)}{n_{j}^{f}} \mathbf{v}_{j} \sum_{i \neq j}^{N_{f}} \frac{w^{f}\left(r_{a i}\right)}{n_{i}^{f}}\right)\right] .
\end{aligned}
$$

Using Eq. (5) and rewriting the last term, we obtain

$$
\begin{aligned}
\mathbf{F}_{a}^{f p}= & \xi\left[\frac{\mathrm{d} \mathbf{r}_{a}^{*}}{\mathrm{~d} t}-\sum_{i=1}^{N_{f}}\left(\frac{w^{f}\left(r_{a i}\right)}{n_{i}^{f}}\right)^{2} \mathbf{v}_{i}\right. \\
& \left.-\sum_{j=1}^{N_{f}} \frac{w^{f}\left(r_{a j}\right)}{n_{j}^{f}} \mathbf{v}_{j}\left(1-\frac{w^{f}\left(r_{a j}\right)}{n_{j}^{f}}\right)\right] .
\end{aligned}
$$

Simplifying further and noting that two of the terms cancel each other and replacing $j$ with $i$, we get

$$
\mathbf{F}_{a}^{f p}=\xi\left(\frac{\mathrm{d} \mathbf{r}_{a}^{*}}{\mathrm{~d} t}-\sum_{i=1}^{N_{f}} \frac{w^{f}\left(r_{a i}\right)}{n_{i}^{f}} \mathbf{v}_{i}\right)
$$

which is clearly equal and opposite to the total force exerted by the fluid on the polymer blob $a$, i.e., $\mathbf{F}_{a}^{p f}$ shown in Eq. (7).

\section{APPENDIX B: DERIVATION OF STOCHASTIC UPDATE FOR THE FLUID VELOCITIES}

In this appendix, we derive the statistical properties of the velocity fluctuations $\mathrm{d} \mathbf{W}_{j k}^{v}$ for the pure fluid at quiescent state. Although we use a different approach, we essentially arrive at the same result as the velocity fluctuations for an incompressible SDPD fluid derived using the GENERIC approach. $^{23,24}$

According to the Chapman-Kolmogorov equation, the probability to find the system at time $t+\mathrm{d} t$ at the point $z$ in the phase space characterised by positions and velocities, i.e., $\mathbf{z}=\left\{\mathbf{r}_{1}, \ldots, \mathbf{r}_{N}, \mathbf{v}_{1}, \ldots, \mathbf{v}_{N}\right\}$, given that at $t=0$ the system was at point $\mathbf{z}_{0}$ in the phase space, is given by

$$
\mathcal{G}\left(\mathbf{z} ; \mathbf{z}_{0} ; t+\mathrm{d} t\right)=\int \mathrm{d}^{6 N} z^{\prime} \mathcal{G}\left(\mathbf{z} ; \mathbf{z}^{\prime} ; \mathrm{d} t\right) \mathcal{G}\left(\mathbf{z}^{\prime} ; \mathbf{z}_{0} ; t\right)
$$

i.e., where $\mathbf{z}^{\prime}$ is any intermediate point in the phase space, through which the system could have passed at time $t$ with $\lim _{\mathrm{d} t \rightarrow 0} \mathcal{G}\left(\mathbf{z} ; \mathbf{z}^{\prime} ; \mathrm{d} t\right)=\delta\left(\mathbf{z}-\mathbf{z}^{\prime}\right)$. Going through the usual derivation, we arrive at the following Fokker-Planck equation:

$$
\begin{aligned}
& \frac{\partial \mathcal{G}\left(\mathbf{z} ; \mathbf{z}_{0} ; t\right)}{\partial t} \\
& =-\sum_{i} \sum_{\alpha} \frac{\partial}{\partial r_{i, \alpha}}\left\{\left(v_{i, \alpha}\right) \mathcal{G}\left(\mathbf{z} ; \mathbf{z}_{0} ; t\right)\right\} \\
& \quad-\sum_{i} \sum_{\alpha} \frac{\partial}{\partial v_{i, \alpha}}\left\{\left(\sum_{j} \frac{f_{i j}}{m}\left(v_{j, \alpha}-v_{i, \alpha}\right)+\frac{F_{i, \alpha}}{m}\right) \mathcal{G}\left(\mathbf{z} ; \mathbf{z}_{0} ; t\right)\right. \\
& \left.\quad-\frac{1}{2} \sum_{j} \sum_{k} \sum_{l} \sum_{\beta} \frac{\partial}{\partial v_{i, \beta}}\left[\frac{\left\langle\mathrm{d} W_{i k, \alpha}^{v} \mathrm{~d} W_{j l, \beta}^{v}\right\rangle}{\mathrm{d} t} \mathcal{G}\left(\mathbf{z} ; \mathbf{z}_{0} ; t\right)\right]\right\},
\end{aligned}
$$

where $\alpha$ and $\beta$ run from 1 to 3 corresponding to the different Cartesian components and $F_{i}$ represents the force due to the pressure gradient term,

$$
\frac{F_{i}}{m}=\frac{(\nabla P)_{i}}{\rho_{i}}=\boldsymbol{\nabla}\left(\frac{P}{\rho}\right)_{i}+\frac{P_{i}}{\rho_{i}^{2}}(\nabla \rho)_{i} .
$$

Since the standard SPH formalism for a gradient of any property A is given by $(\nabla A)_{i}=\sum_{j=1}^{N} \frac{m_{i} A_{i}}{\rho_{i}} \frac{\mathrm{d} w}{\mathrm{~d} r}\left(r_{i j}\right) \frac{\mathbf{r}_{i j}}{r_{i j}}$, we get

$$
F_{i}=\sum_{j=1}^{N_{f}}\left(\frac{P_{j}}{\left(n_{j}^{f}\right)^{2}}+\frac{P_{i}}{\left(n_{i}^{f}\right)^{2}}\right) \frac{\mathrm{d} \omega^{f}}{\mathrm{~d} r}\left(r_{i j}\right) \frac{\mathbf{r}_{i j}}{r_{i j}},
$$

which is the form that has been shown in Eq. (11) and used thereafter in Eq. (16).

At steady state, we expect the left-hand side of Eq. (B2) to be zero. Furthermore, we expect that for a system in quiescent state, the equilibrium distribution of $\mathbf{z}$ is given by the MaxwellBoltzmann distribution

$$
\mathcal{G}^{e q}(\mathbf{z}) \propto \exp \left\{\frac{1}{k_{B} T} \Phi\left(\mathbf{r}_{1}, \ldots, \mathbf{r}_{N}\right)-\frac{1}{2 k_{B} T} \sum_{i} m \mathbf{v}_{i} \cdot \mathbf{v}_{i}\right\}
$$

where $\Phi$ is the total potential energy based on the pressure distribution of the system given by $\Phi=P V=\sum_{i=1}^{N_{f}} P_{i} V / N_{f}$, where $N_{f} / V$ may be replaced by $\bar{n}^{f}$, from which the conservative forces can be calculated as $\mathbf{F}_{i}=-\partial \Phi / \partial \boldsymbol{r}_{i}$. At equilibrium, $\mathcal{G}\left(\mathbf{z} ; \mathbf{z}_{0} ; t\right)$ in Eq. (B2) must be replaced by $\mathcal{G}^{e q}(\mathbf{z})$.

We pre-calculate some of the derivatives that will be required as follows:

$$
\begin{aligned}
\frac{\partial}{\partial \mathbf{r}_{i}} \mathcal{G}^{e q}(\mathbf{z}) & =-\frac{1}{k_{B} T}\left(\frac{\partial}{\partial \mathbf{r}_{i}} \Phi\right) \mathcal{G}^{e q}(\mathbf{z})=\frac{\mathbf{F}_{i} \mathcal{G}^{e q}(\mathbf{z})}{k_{B} T}, \\
\frac{\partial}{\partial \mathbf{v}_{i}} \mathcal{G}^{e q}(\mathbf{z}) & =-\frac{m \mathbf{v}_{i} \mathcal{G}^{e q}(\mathbf{z})}{k_{B} T}
\end{aligned}
$$

Notice that these imply

$\sum_{i} \sum_{\alpha} \frac{\partial}{\partial r_{i, \alpha}} v_{i, \alpha} \mathcal{G}^{e q}(\mathbf{z})+\sum_{i} \sum_{\alpha} \frac{\partial}{\partial v_{i, \alpha}} \frac{F_{i, \alpha}}{m} \mathcal{G}^{e q}(\mathbf{z})=0$.

Therefore, these terms will get eliminated from Eq. (B2) at equilibrium. As a result, all remaining sums between curly brackets in Eq. (B2) with $\mathcal{G}\left(\mathbf{z} ; \mathbf{z}_{0} ; t\right)$ replaced by $\mathcal{G}^{e q}(\mathbf{z})$ must be identically equal to zero.

We re-write the correlations of the velocity fluctuations as follows:

$$
\left\langle\mathrm{d} W_{i k, \alpha}^{v} \mathrm{~d} W_{j l, \beta}^{v}\right\rangle=2 k_{B} T C_{i k \alpha j l \beta}^{v v} \mathrm{~d} t .
$$


Thus, at equilibrium, we must have

$$
\sum_{j} \frac{f_{i j}}{m}\left(v_{j, \alpha}-v_{i, \alpha}\right)+\sum_{j} \sum_{k} \sum_{l} \sum_{\beta} C_{i k \alpha j l \beta}^{v v} m v_{i, \beta}=0 .
$$

These equations will be identically satisfied if we choose $C_{i k \alpha j l \beta}^{v v}=\delta_{\alpha \beta}\left[\left(f_{i k} / m^{2}\right) \delta_{i j} \delta_{k l}-\left(f_{k i} / m^{2}\right) \delta_{i l} \delta_{j k}\right]$.

Thus, in conclusion, to get the equilibrium distribution at steady state, we must choose the stochastic variables according to

$$
\begin{aligned}
\left\langle\mathrm{d} \mathbf{W}_{i j}^{v} \mathrm{~d} \mathbf{W}_{i j}^{v}\right\rangle & =\left(\frac{2 k_{B} T}{m}\right)\left(\frac{\mathrm{d} t}{m}\right) f_{i j} \mathbf{I}, \\
\left\langle\mathrm{d} \mathbf{W}_{i k}^{v} \mathrm{~d} \mathbf{W}_{j l}^{v}\right\rangle & =\mathbf{0} \quad(i k \neq j l \wedge i k \neq l j) .
\end{aligned}
$$

${ }^{1}$ B. Dünweg and K. Kremer, Phys. Rev. Lett. 66, 2996 (1991).

${ }^{2}$ B. Dünweg and K. Kremer, J. Chem. Phys. 99, 6983 (1993).

${ }^{3}$ P. Ahlrichs and B. Dünweg, J. Chem. Phys. 111, 8225 (1999).

${ }^{4}$ A. Malevanets and R. Kapral, J. Chem. Phys. 110, 8605 (1999).

${ }^{5}$ A. Malevanets and R. Kapral, J. Chem. Phys. 112, 7260 (2000).

${ }^{6}$ A. Malevanets and J. Yeomans, Europhys. Lett. 52, 231 (2000).

${ }^{7}$ M. Ripoll, K. Mussawisade, R. Winkler, and G. Gompper, Europhys. Lett. 68, 106 (2004).

${ }^{8}$ R. Kapral, Adv. Chem. Phys. 140, 89 (2008).

${ }^{9}$ G. Gompper, T. Ihle, D. Kroll, and R. Winkler, Adv. Polym. Sci. 221, 1 (2009).

${ }^{10}$ C.-C. Huang, R. G. Winkler, G. Sutmann, and G. Gompper, Macromolecules 43, 10107 (2010).

${ }^{11}$ P. J. Hoogerbrugge and J. M. V. A. Koelman, Europhys. Lett. 19, 155 (1992).

12 J. M. V. A. Koelman and P. J. Hoogerbrugge, Europhys. Lett. 21, 363 (1993).

${ }^{13}$ P. Espanol and P. Warren, Europhys. Lett. 30, 191 (1995).

${ }^{14}$ Y. Kong, C. Manke, W. Madden, and A. Schlijper, Int. J. Thermophys. 15, 1093 (1994).

${ }^{15}$ A. Schlijper, P. Hoogerbrugge, and C. Manke, J.Rheol. 39, 567 (1995).

${ }^{16}$ X. Fan, N. Phan-Thien, N. T. Yong, X. Wu, and D. Xu, Phys.Fluids 15, 11 (2003).

${ }^{17}$ X. Fan, N. Phan-Thien, S. Chen, X. Wu, and T. Yong Ng, Phys. Fluids 18, 063102 (2006).

${ }^{18}$ J. P. Hernández-Ortiz, H. Ma, J. J. de Pablo, and M. D. Graham, Phys. Fluids 18, 123101 (2006).

${ }^{19}$ J. A. Millan, W. Jiang, M. Laradji, and Y. Wang, J. Chem. Phys. 126, 124905 (2007).

${ }^{20}$ D. A. Fedosov, G. Em Karniadakis, and B. Caswell, J. Chem. Phys. 128, 144903 (2008).

${ }^{21}$ J. A. Millan and M. Laradji, Macromolecules 42, 803 (2009).
${ }^{22}$ Z. Li, Y. Li, Y. Wang, Z. Sun, and L. An, Macromolecules 43, 5896 (2010).

${ }^{23}$ P. Espanol and M. Revenga, Phys. Rev. E 67, 026705 (2003).

${ }^{24}$ A. Vázquez-Quesada, M. Ellero, and P. Español, J. Chem. Phys. 130, 034901 (2009).

${ }^{25}$ L. B. Lucy, Astron.J. 82, 1013 (1977).

${ }^{26}$ R. A. Gingold and J. J. Monaghan, Mon. Not. R. Astron. Soc. 181, 375 (1977).

${ }^{27}$ R. Gingold and J. Monaghan, J. Comput. Phys. 46, 429 (1982).

${ }^{28}$ J. J. Monaghan, Annu. Rev. Astron. Astrophys. 30, 543 (1992).

${ }^{29}$ J. J. Monaghan, Rep. Prog. Phys. 68, 1703 (2005).

${ }^{30}$ S. Litvinov, M. Ellero, X. Hu, and N. A. Adams, Phys. Rev. E 77, 066703 (2008).

${ }^{31}$ S. Litvinov, Q. Xie, X. Hu, N. Adams, and M. Ellero, Fluids 1, 7 (2016).

${ }^{32}$ P. Español and P. B. Warren, J. Chem. Phys. 146, 150901 (2017).

${ }^{33}$ A. Van den Noort, W. K. den Otter, and W. J. Briels, Europhys. Lett. 80, 28003 (2007).

${ }^{34}$ W. J. Briels, Soft Matter 5, 4401 (2009).

${ }^{35}$ I. S. Santos de Oliveira, A. van den Noort, J. T. Padding, W. K. den Otter, and W. J. Briels, J. Chem. Phys. 135, 104902 (2011).

${ }^{36}$ I. S. Santos de Oliveira, W. K. den Otter, and W. J. Briels, J. Chem. Phys. 137, 204908 (2012).

${ }^{37}$ I. S. Santos de Oliveira, B. W. Fitzgerald, W. K. den Otter, and W. J. Briels, J. Chem. Phys. 140, 104903 (2014).

${ }^{38}$ J. T. Padding and W. J. Briels, J. Chem. Phys. 141, 244108 (2014).

${ }^{39}$ V. R. Ahuja, J. van der Gucht, and W. J. Briels, J. Chem. Phys. 145, 194903 (2016).

${ }^{40}$ W. J. Briels, Theory of Polymer Dynamics (Lecture Notes, Uppsala, Sweden, 1994).

${ }^{41}$ C. W. Gardiner et al., Handbook of Stochastic Methods (Springer Berlin, 1985), Vol. 4.

${ }^{42}$ L. Landau and E. Lifshitz, Statistical Physics (Pergamon Press, 1958).

${ }^{43}$ L. Landau and E. Lifshitz, Fluid Mechanics (Pergamon Press, 1959).

${ }^{44}$ J. M. O. De Zárate and J. V. Sengers, J. Stat. Phys. 115, 1341 (2004).

${ }^{45}$ J. M. O. De Zárate and J. V. Sengers, Hydrodynamic Fluctuations in Fluids and Fluid Mixtures (Elsevier, 2006).

${ }^{46}$ B. Hoomans, J. Kuipers, W. J. Briels, and W. P. M. van Swaaij, Chem. Eng. Sci. 51, 99 (1996).

${ }^{47}$ G. K. Batchelor, An Introduction to Fluid Dynamics (Cambridge University Press, 2000).

${ }^{48}$ R. H. Cole, Underwater Explosions (Dover Publications, 1965).

${ }^{49}$ J. P. Boon and S. Yip, Molecular Hydrodynamics (Courier Corporation, 1980).

${ }^{50}$ A. Lees and S. Edwards, J. Phys. C: Solid State Phys. 5, 1921 (1972).

${ }^{51}$ D. A. Fedosov, G. E. Karniadakis, and B. Caswell, J. Chem. Phys. 132, 144103 (2010).

${ }^{52}$ R. Bird, W. Stewart, and E. Lightfoot, Transport Phenomena, Wiley International Edition (Wiley, 2007).

${ }^{53} \mathrm{Comsol}$, Multiphysics User Guide for COMSOL 5.0, 2014

${ }^{54}$ L. Fang, H. Hu, and R. G. Larson, J. Rheol. 49, 127 (2005). 